NELLY NAUMANN

\title{
Der Tiger in chinesischen Märchen, Sagen und frühen religiösen Vorstellungen
}




\section{Der Tiger in chinesischen Märchen, Sagen und frühen religiösen Vorstellungen}

Der Tiger, das mächtigste Raubtier auf dem asiatischen Kontinent, hat selbstverständlich einen festen Platz in den chinesischen Tier- und Schwankmärchen, wenn er dort auch keine besonders wichtige oder gar rühmliche Rolle spielt ${ }^{1}$. Sein wirkliches Wesen kommt im Zaubermärchen zum Vorschein.

In ganz China kennt man das Märchen von der Mutter, die eines Tages die Großmutter besuchen wollte und unterwegs vom Tiger gefressen wurde.

Die Frau ermahnt ihre Kinder, niemanden einzulassen; am Nachmittag wolle sie zurück sein. Es ist bereits dunkel, als der Tiger, der dies beobachtet, die Mutter gefressen und ihre Kleider angezogen hat, bei den Kindern Einlaß begehrt. Die Kinder sind vorsichtig, die Stimme scheint nicht die der Mutter, doch am Ende öffnen sie der falschen Mutter die Tür. Alle gehen zu Bett, aber die größeren Kinder bleiben wach und merken, daß die Mutter etwas ißt. Sie sind hungrig, und da sie nicht aufhören zu betteln, wirft ihnen die Mutter am Ende ein Rübchen hin - doch das ist ein Finger ihres Brüderchens. Nun ahnen sie, wer die Mutter ist und was sie erwartet, wenn sie bleiben. So verlangen sie hinaus, um ein Geschäftchen zu machen. Sie klettern auf einen hohen Baum, und als der Tiger ihnen nachgeht und fragt, warum sie da oben sitzen, machen sie ihm weis, etwas ganz Besonderes zu sehen. Er soll sich nur in einen Korb setzen, den wollen sie an einem Seil hochziehen. Sie lassen das Seil zur rechten Zeit los, der Tiger bricht sich das Genick ${ }^{2}$.

Dies ist eine von mehreren Versionen - manchmal ist ein anderer Schluß angehängt. Doch das Wesentliche bleibt sich immer gleich: Der Tiger, der die Mutter und meist noch das Brüderchen gefressen hat, kommt zu Tode.

Das Märchen ist weit über China hinaus bekannt. Es wird auch in der Mand-

1 cf. Eberhard, W.: Typen chinesischer Volksmärchen (FFC 120). Helsinki 1937, Typ 3: Der Tiger und der Hirsch, Typ 4: Der Tiger und der Esel (=Ting, N.-t.: A Type Index of Chinese Folktales [FFC 223]. Helsinki 1978, Typ 78: Animal Allows Himself to be Tied to Another for Safety, Typ 78 B: The Monkey is Tied to the Tiger; cf. Eberhard, W.: Volksmärchen aus Südost-China [FFC 128]. Helsinki 1941, num. 2); Ting op. cit., Typ 157: Learning to Fear Man; Eberhard, op. cit. 1937, Typ 10: Die Angst vor der Traufe (=Ting op. cit., Typ 177: The Thief and the Tiger; cf. Eberhard op. cit. 1941, num.7); Ting op. cit., Typ $101 \%$ : The Dog Wants to Imitate the Wolf; ibid., Typ $159 \mathrm{~A}_{1}$ : The Tiger Swallows Red Hot Iron.

2 Eberhard 1937 (wie not. 1) Typ 11: Der Tiger und die Kinder; id. 1941 (wie not. 1) num. 8; Ting (wie not. 1) Typ 333 C: The Tiger Grandma; cf. Lessing, B.: Fünf chinesische Volksmärchen aus Schantung. In: Sinica 10, 3 (1935) 110-112; Wilhelm, R.: Chinesische Volksmärchen (Die Märchen der Weltliteratur). Jena 1914, num.9. 
schurei und in Korea erzählt ${ }^{3}$; in Japan, wo es keine Tiger gibt, tritt an die Stelle des Tigers eine Menschenfresserin ${ }^{4}$. Auch in chinesischen Varianten kann ein Panther, Wolf oder Bär oder eine Menschenfresserin die Stelle des Tigers einnehmen. Manchmal klettern die Kinder vom Baum geradewegs in den Himmel, von wo ihnen eine Kette herabgelassen wird, dem bösen Dämon jedoch ein verrottetes Seil, so daß er zu Tode stürzt. Im koreanischen Märchen werden die beiden Kinder zu Sonne und Mond ${ }^{5}$.

Hat man dieses Märchen nur erzählt, um die Kinder zur Vorsicht zu ermahnen? Die weite Verbreitung des Märchens, dessen Grundzüge trotz der vielen Varianten stets dieselben bleiben, hat gewiß mehr zu bedeuten. In seinem Mittelpunkt steht der Tiger, auch wenn er durch ein anderes großes Raubtier oder, die Menschenfresserin' ersetzt werden kann. Dieser Tiger ist jedoch kein gewöhnlicher Tiger, so wie auch die Menschenfresserin keine gewöhnliche Frau ist. Er ist ein Tigerdämon, der sich in einen Menschen verwandeln kann.

Allerdings kann sich der Tiger auch von einer ganz anderen Seite zeigen. $\mathrm{Da}$ wird auch erzählt, wie eine Tigerin zum Dank für Hebammendienste bei einer schweren Geburt der Helferin eine Schwiegertochter ins Haus bringt; dem Mann, der einem verwundeten Tiger beistand, rettet der Tiger das Leben oder hilft ihm bei einem Feldzug; einen Mann, der in eine Tigerhöhle gefallen war und die Jungen fütterte, belohnt die Tigermutter mit Geld ${ }^{6}$. In einem weiteren Märchen kommt in das Haus eines einsamen Mannes eine Frau. Es ist eine Tigerin, die ihr Fell abgelegt hat. Der Mann oder jemand anderes versteckt es, so bleibt die Frau ein Mensch. Nach langer Zeit gerät sie wieder in den Besitz des Fells und läuft als Tier davon - nicht ohne gelegentlich vorher noch gewisse Leute zu verspeisen'. Schließlich ist das nur in Kuangtung verbreitete Märchen von der Tigermutter zu erwähnen, die ein Mädchen als Tochter aufzieht. Ein Beamter entdeckt das Mädchen und heiratet es. Die Tigermutter findet den Weg zu dem Mädchen durch ausgeworfene Stäbchen, doch sie kommt um. Als Anfügung zu

3 cf. Eberhard 1937 (wie not. 1) 20-23; Choi, I.-h.: A Type Index of Korean Folktales. Seoul 1979, Typ 100: The Origin of the Sun and the Moon.

4 Ikeda, H.: A Type and Motif Index of Japanese Folk-Literature (FFC 209). Helsinki 1971, Type 333 A: The Gluttonous Ogress and the Children; cf. Hammitzsch, H. (ed.): Japanische Volksmärchen (Die Märchen der Weltliteratur). Düsseldorf/Köln 1964, num.20; v. auch Naumann, N.: Verschlinger Tod und Menschenfresser. Zur Wandlung eines mythischen Bildes im japanischen Märchen. In: Saeculum 22, 1 (1971) 59-70, hier 66.

5 Choi (wie not. 3); cf. Chun, Sh.-y.: Korean Folk Tales. Seoul 1979, 29-31.

6 Eberhard 1937 (wie not. 1) Typ 17: Der Dank des Tigers (=Ting [wie not.1] Typ 156: Thorn Removed from Lion's Paw, Typ 156 B *: Woman as Snake's Midwife; cf. Eberhard 1941 [wie not. 1] num. 12). Ein weiteres Beispiel, das in diesen Zusammenhang gehört, ist Ting, Typ 156D*: The Noble Tiger: "A tiger has eaten the only son of an old woman, who goes to court to sue the tiger for killing the only breadwinner in her family. The magistrate issues a subpoena, and a drunk constable delivers it to the forest. When the tiger hears about it, he comes voluntarily to the court and admits his own guilt. He also supplies the old woman with food, etc., until her death."

7 Eberhard 1937 (wie not. 1) Typ 37: Die Tigerfrau; id. 1941 (wie not. 1) num. 24. 
diesem Märchen erwähnt Wolfram Eberhard: „Tochter bekommt Kind, das ihr von den anderen Nebenfrauen weggenommen wird. Sie wird verstossen. Durch Traum, den der tote Tiger gibt, kommt alles ans Licht, die Nebenfrauen werden getötet, die Tochter rechte Frau. " 8

Man muß den Blick auf die Sage lenken, um zu erkennen, daß in diesen Märchen außerordentlich alte Vorstellungen weiterleben. So ist der älteste Beleg für die Tigermutter, die sich eines Menschenkindes annimmt, schon in einem noch in vorchristlicher Zeit entstandenen historischen Werk, dem Tso-chuan, zu finden, einem Kommentar zum Ch'un-ch'iu (Frühling[s]- und Herbst[-Annalen]) ${ }^{9}$. Im Tso-chuan wird berichtet, daß Tou Po-pi (790-764 v. Chr.), der Sohn des Fürsten Jo-ao von Ch'u, heimlich mit der Tochter des Freiherrn von Yün verkehrte. Diese gebar den Tzu-wen. Die Freifrau von Yün ließ das Kind in Meng aussetzen. Eine Tigerin säugte es. Als der Freiherr von Yün dort jagte, sah er sie. Er erschrak und kehrte um. Nun berichtete ihm seine Gattin, was geschehen war. Daraufhin schickte er jemanden und nahm das Kind an. Das Kind wurde tatsächlich ,der Ministerpräsident Tzu-wen'. Der von einer Tigerin gesäugte Tzu-wen ist der Ahn der Familie Pan, was ,gefleckt ${ }^{c}$ bedeutet ${ }^{10}$.

Andere Sagen oder Mythen bringen den Tiger in noch engere Verbindung mit dem Ahnherrn einer bestimmten Familie. Das Hou-Han-shu, die Geschichte der Späteren Han-Dynastie (25-220 n. Chr.), zeigt den Tiger als Schutz-oder Ahntier der Ch'iang ${ }^{11}$. Deutlicher noch als in der Herkunftssage der Pan und der Ch'iang ist Lin-chün, der Ahn der $\mathrm{Pa}$, selbst als Tiger gekennzeichnet: „Als er starb, verwandelten sich seine hun- und seine p'o-Seele und wurden für immer zu einem weißen Tiger; weil Tiger Menschenblut trinken, bringt der $\mathrm{Pa}-\mathrm{Clan}$ diesem Menschenopfer dar. “ 12

Auch für die Geschichte von der zur Menschenfrau verwandelten Tigerin, die Kinder zur Welt bringt, liegt ein früher Beleg aus der T'ang-Zeit (618-905) $\operatorname{vor}^{13}$. J.J.M. de Groot, der mehrere entsprechende Texte anführt, prangert geradezu diejenigen als die schlimmsten Tigerdämonen an, die in bösartiger Absicht die Gestalt einer Frau annehmen und Männer dazu bringen, sie zu heira-

8 id. 1937 (wie not. 1) Typ 120: Die Tiger-Mutter; cf. auch Ting (wie not. 1) Typ 535: The Boy Adopted by Tigers: „I.( $\left.b^{1}\right)$ The boy's father has become a weretiger.[...] II.(a) The tiger arranges the marriage by carrying the girl to the hero. (g) When he is accused of kidnapping her, the tiger comes to the court to bear witness and the man is released."

9 Tso-chuan. Ch'un-ch'iu tso-chuan, Fasz. 21 (Shih-san-ching chu-su 7 [t. 27-32]). Peiching 1957,873 sq.

10 cf. Erkes, E.: Der ikonographische Charakter einiger Chou-Bronzen. 1: Der Tiger. In: Artibus Asiae 6 (1936) 111-117, hier 112; Eberhard, W.: Lokalkulturen im alten China. 1: Die Lokalkulturen des Nordens und Westens (T'oung Pao. Suppl. zu t. 37). Leiden 1942, 344.

11 Hou-Han-shu. Shanghai ${ }^{2} 1973$, Fasz. 87, 2875; cf. Eberhard (wie not. 10) 345.

12 Hou-Han-shu (wie not. 11) Fasz. 86, 2840; cf. Groot, J. J. M. de: The Religious System of China. 4,2,1: The Soul in Philosophy and Folk-Conception. Leiden 1901, 167 sq.; Eberhard (wie not. 10) 344-346.

13 id. 1941 (wie not. 1) 65 . 
ten, nur um am Ende diese samt den inzwischen erzeugten Kindern zu verschlingen $^{14}$.

Tigergeschichten handeln jedoch nicht nur von Tigern, die sich in Menschen verwandeln. Es gibt auch Menschen, die sich ihrerseits in Tiger verwandeln können. Der älteste Bericht über eine solche Verwandlung ist Liu An (†122 v. Chr.) zu danken; in dem von ihm verfaßten Werk Huai-nan-tzu heißt es: „Einen Mann namens Kung-niu Ai befiel eine Krankheit und am siebten Tag verwandelte er sich in einen Tiger. Sein Bruder hatte die Tür verschlossen, und als er hineinging, um nach ihm zu sehen, ergriff ihn der Tiger und tötete ihn. Musterung und Farbe hatten ihn zum Tier gemacht, Nägel und Zähne waren andere geworden, Neigung und Herz waren verwandelt, und mit der Gestalt hatte sich auch sein Geist verändert. So kann man nicht wissen, ob das, was jetzt ein Tiger ist, vorher ein Mensch war, oder was jetzt ein Mensch ist, vorher ein Tiger war." Kao Yu, ein Kommentator des 2. Jahrhunderts n. Chr., meinte hierzu, Wahnsinnige hätten periodische Anfälle, und „wenn einer dabei zu einem Tiger wird, dann frißt er eben auch Menschen. Hat einer nun Menschen gefressen, so wird er ein wirklicher Tiger. Wer aber keinen Menschen gefressen hat, der verwandelt sich wieder zurück zu einem Menschen. " ${ }^{15}$ Dies war nicht etwa lediglich primitiver Aberglaube einfacher Leute. Selbst der große Skeptiker Wang Ch'ung (27-97) hält diese Geschichte für wahr; gleichzeitig spricht er in den von ihm verfaßten ,Kritischen Abhandlungen' des Lun-heng davon, daß das ching, der Geist oder die Essenz alter Tiere, sich in einen Menschen verwandeln kann; es gebe aber auch solche, die das von Natur aus vermögen, auch ohne alt zu sein ${ }^{16}$.

So kennt man offenbar zweierlei Arten von Wer-Tigern: Menschen, die sich in Tiger verwandeln, und Tiger, die sich in Menschen verwandeln. Da darf es nicht wundern, daß seit alters viele Geschichten in Umlauf waren, die von Wer-Tigern der einen wie der anderen Art erzählten. Manchmal heißt es, man könne WerTiger daran erkennen, daß sie bestimmte Merkmale besäßen; Tiger können zum Beispiel ihren Schwanz nicht mitverwandeln. Wollte einer voll und ganz zum Menschen werden, mußte der Schwanz verbrannt werden. Wer-Tiger wiederum, die eigentlich Menschen sind, muß man bei ihrem Namen nennen, dann laufen sie davon.

De Groot wies darauf hin, daß die vielen Tigergeschichten in den Büchern aller Zeiten ein Zeichen dafür sind, wie stark der Glaube an die Tigerdämonen war. Ja, der Glaube sei auch jetzt keineswegs ausgerottet, und wenn man die Chinesen näher kenne, könne man sich sehr bald überzeugen, daß sie das über Tiger Erzählte als Tatsache nehmen. Dies kommt vor allem in den vielen Sagen zum Aus-

14 Groot, J.J. W. de: The Religious System of China. 4,2,2: Demonology. Leiden 1907, 550.

15 Huai-nan-tzu. Kao Yu chu (Chu-tzu chi-ch'eng 7). Pei-ching ${ }^{4} 1959$, Fasz. 2, 20; cf. de Groot (wie not. 12) 160 sq.

16 Lun-heng (Chu-tzu chi-ch'eng 7). Pei-ching ${ }^{4} 1959$, 202: Lun ssu p'ien; ibid., 220: Ting kuei p'ien; cf. de Groot (wie not. 12) 161 sq. 
druck, in denen von namentlich genannten Menschen die Rede ist, die sich in Tiger verwandeln können. Sind sie einmal erkannt, kann man sie den Behörden melden und zu Tode jagen. So fragt er denn weiterhin im Zusammenhang mit den von ihm angeführten Geschichten, wie viele Unglückliche wohl verdächtigt und unschuldig diesem Wahn zum Opfer gefallen seien ${ }^{17}$. Der Glaube an Wer-Tiger, der durchaus dem europäischen Werwolfglauben entspricht, ist freilich nicht auf China beschränkt; es darf daher nicht wundern, daß die Chinesen schon in alter Zeit glaubten, gewisse südchinesische Fremdstämme besäßen generell die Fähigkeit, sich in Tiger zu verwandeln ${ }^{18}$.

Die Verwandlung vom Menschen zum Tiger kann durch ein Zaubermittel geschehen ${ }^{19}$; meist wird sie jedoch durch Anlegen einer Tigerhaut bewirkt. Nicht immer geschieht die Verwandlung freiwillig, sie kann auch ein böser Zauber oder eine göttliche Strafe sein. Zwar erscheinen die Wer-Tiger vor allem als raubgierige Bestien, doch es gibt Ausnahmen. So legt ein zur Strafe verwandelter WerTiger immer wieder Wild vor die Tür seiner Verwandten. Eines Tages kommt er gar zurück, wieder Mensch geworden. Nur sein Kopf blieb unverwandelt und so versetzt er alle so sehr in Schrecken, daß man ihn vertreibt ${ }^{20}$. Eine Geschichte, die N. Dennys ${ }^{21}$ anführt, verweist auf den Tiger als göttliches Wesen:

Ein Reisender, der sich verspätet hatte, bemerkte im Wald ein hellglänzendes Licht und einen Mann in roter Kleidung mit goldener Krone und erlesener Rüstung, vor und hinter ihm zahlreiches Gefolge. Der Reisende hielt sich klugerweise verborgen. Als er am nächsten Tag die Leute in der Umgebung nach dem vornehmen Herrn befragte, sagten sie ihm, das sei der Tigergeist der Berge. „Sobald er Menschen verschlingen will, legt er seine Kleider ab und wird zum Tiger, stürzt sich auf den Wanderer und reißt ihn in Stücke. Ihr seid dem auf wunderbare Weise entgangen!“

Kult- und Opferplätze, auch Tempel, in denen der Gott als Tiger auftritt, gibt es nach Eberhard an mehreren Orten in Hupeh und Kuanghsi; für Kuichou spricht er von Opferplätzen für den „Ehevermittler Tiger“. Er schließt hier die Bemerkung an, daß „für dieses Gebiet die Märchen von der Verwandlung eines Men-

17 de Groot (wie not. 14) 544-546.

18 cf. Eberhard, W.: Kultur und Siedlung der Randvölker Chinas (T'oung Pao. Suppl. zu t. 36). Leiden 1942, 214.

19 de Groot (wie not. 12) 169.

20 ibid., 176 sq.; cf. auch ibid., 179: Der Tiger bringt seinen Verwandten Schweine! Hier sei generell auf die zahlreichen Texte über Tigerdämonen und Wer-Tiger verwiesen, die de Groot (ibid., 160-182; id. [wie not. 14] 544-563) zusammengestellt hat. In einem schwankartigen koreanischen Märchen trifft ein Holzfäller im Wald auf einen hungrigen Tiger und begrüßt ihn furchtlos als, seinen Bruder'. Der Tiger läßt sich von dem Mann überzeugen - vielleicht war er wirklich ursprünglich ein Mensch? So entgeht der Holzfäller der Gefahr, der Tiger aber legt von nun an immer wieder Wild in den Garten des Holzfällers (Choi [wie not. 5] Typ 116: The Tiger's Brotherhood). Das in Huwe, A.: Korea (Märchen aus aller Welt 16). München 1979, 38-42 übersetzte Märchen „Bruder Tiger“ besitzt einen erweiterten Schluß.

21 Dennis, N. B.: The Folklore of China and Its Affinities with That of the Aryan and Semitic Races [1876]. Reprint Amsterdam 1968, 91. 
schen in einen Tiger, der Menschen frißt, aber auch heiratet und nur zeitweise Tiger ist, typisch sind“" 22 . Märchen und Sage ergeben somit ein eigenartiges Bild. Vom Tiger ist die Rede, aber ist dieser Tiger wirklich ein Tiger? Ist er etwa nur ein verwandelter Mensch? Ist er ein Dämon, oder gar ein Gott? Ist er von Grund auf eine reißende Bestie? Oder ist diese Rolle nur vorgetäuscht?

Tatsächlich ist der Tiger im Volksglauben keineswegs nur das gefährliche Raubtier, er ist vor allem anderen dasjenige Wesen, das die bösen Geister abwehrt und verschlingt. Daher setzt man (vermutlich auch heute noch) den kleinen Kindern Mützchen auf, die einen Tigerkopf darstellen. Noch bis in unsere Zeit hat man zum Jahreswechsel an die Türpfosten die Bilder zweier Geister, Shen-t'u und Yü-lü, aufgeklebt, dazu das Bild des Tigers. Der bereits erwähnte Wang Ch'ung beschreibt in seinem Werk Lun-heng die Sitte für das erste nachchristliche Jahrhundert. Damals schnitzten die Präfektur-Beamten die Figuren aus Pfirsichholz und malten den Tiger auf Türen und Tore. Zur Erläuterung führt er eine verlorengegangene Stelle des Shan-bai-ching (Klassiker der Berge und Meere) an, einer descriptio mundi, deren älteste Teile vermutlich noch vor $250 \mathrm{v}$. Chr. entstanden sind. Diese Passage lautet: „Inmitten des blauen (östlichen) Meeres erhebt sich der Berg Tu-shuo. Darauf steht ein großer Pfirsichbaum, der sich verschlungen über 3000 Meilen hinweg erstreckt. Eine Öffnung seiner Äste im Nordosten heißt Totengeister/Dämonentor. Dort gehen die zehntausend Totengeister ein und aus. Darüber befinden sich zwei göttliche Personen, Shen-t'u und Yü-lü mit Namen, denen die Bewachung der zehntausend Totengeister obliegt. Die bösen und schädlichen greifen sie, binden sie mit Schilfseilen und werfen sie den Tigern zum Fraß vor. Deshalb schuf Huang-ti [der, Gelbe Kaiser'] ein Ritual zu deren jahreszeitlicher Vertreibung, bei dem man große PfirsichholzFiguren aufstellt. Man malt Shen-t'u und Yü-lü sowie den Tiger auf Tore und Türen, man hängt Schilfseile auf und wehrt die bösen Dämonen ab. “23

In den Jahrhunderten vor und nach Christi Geburt begegnet man dem Tiger aber auch in ganz anderem Zusammenhang. So tritt er in der Grabkunst der HanZeit als Begleittier der Hsi-wang-mu auf, der Königinmutter des Westens. Er ist ursprünglich nicht nur ihr Begleittier, er ist eine Erscheinungsform der Hsiwang-mu. Hsi-wang-mu oder Hsi-mu (Mutter des Westens), wie sie auf Orakelknochen genannt wird, ist eine Gottheit, die wiederum aufs engste mit dem Mond und mit aller zum Mond gehörigen Symbolik von Tod und Wiedergeburt verbunden ist. Ihr Sitz ist der K'un-lun-Berg, ein Weltberg. Dort, im Westen

(wie not.16) 221: Ting kuei p'ien; cf. Bodde, D.: Festivals in Classical China. New Year and Other Annual Observances during the Han Dynasty, 206 B.C. A.D. 220. Princeton 1975, 128. Bodde zitiert (not. 160) neben dem Lun-heng eine ganze Reihe Han-zeitlicher Texte, welche die im Shan-hai-ching selbst verlorengegangene Stelle anführen; cf. auch Antoni, K.: Der weiße Hase von Inaba. Vom Mythos zum Märchen (Münchener Ostasiatische Studien 28). Wiesbaden 1982, 207; Münke, W.: Die klassische chinesische Mythologie. Stuttgart 1976, 262 sq. 
Chinas, befindet sich sowohl der Garten der Hsi-wang-mu mit den Pfirsichen des ewigen Lebens, wie auch die Totenwelt ${ }^{24}$.

Der Tiger allerdings vertritt den Westen aus eigenem Recht. So heißt es in dem im 1.Jahrhundert n. Chr. verfaßten Shuo-wen chieb-tzu (Erläuterung der Schriftzeichen) über den Tiger: „Er ist ein Tier der Westgebiete. Man nennt ihn Herr der Vierfüßler. Da er der Herr über die Tiere der Berge ist, nennt man ihn auch Herr der Berge. " ${ }^{25}$ Genau so zeigt ihn noch die oben angeführte Erzählung von der Begegnung des Reisenden mit dem ,Tigergeist der Berge'. Da nun auch die Totenwelt im Westen liegt, scheint es ganz natürlich, daß der Tiger auch als Gott der Toten angesehen wurde ${ }^{26}$. In Korea gilt der Tiger bis heute als die Erscheinungsform des Berggottes; der Berggott aber ist ein Schicksalsgott, der über Tod und Leben entscheidet ${ }^{27}$.

Doch bleiben wir in China. Hinter den eben erwähnten, bereits stark ausgeschmückten und verwässerten Vorstellungen steht letztlich die altchinesische Astralsymbolik, für die der Weiße Tiger - gleichzeitig ein Sternbild - das Tier des Westens ist. Er steht für den Herbst, für Untergang, Verfall, Dunkelheit ${ }^{28}$ und gilt dennoch als ein Yang-Wesen. Sein Gegenspieler, der Blaugrüne Drache (auch er gleichzeitig ein Sternbild), der den Osten und somit den Frühling, das Wiedererstehen der Natur, Licht und Leben vertritt, soll dagegen ein Yin-Wesen sein. Ein späterer Kommentator, Hsü Shen (30?-124?), versuchte, den Widerspruch aufzulösen. Er erklärt, der Tiger sei ein Yang-Tier innerhalb des Yin, der Erde; der Drache sei ein Yin-Reptil innerhalb des Yang, des Himmels ${ }^{29}$. Damit klingt auch die Vorstellung an, daß im Yang das Yin als Potentialität schon vorgebildet oder eingeschlossen ist, im Yin entsprechend das Yang.

Wie alt diese Vorstellungen tatsächlich sind, hat 1987 eine Ausgrabung in Honan ans Licht gebracht. In Hsi-shui-p'o im Kreis P'u-yang fand man eine merkwürdige Grabstätte, die der Yang-shao-Kultur des 4. Jahrtausends v. Chr. zuzurechnen ist. Im Zentrum, mit dem Kopf nach Süden, liegt das Skelett eines Mannes im besten Alter; zu seiner Rechten, d.h. im Osten, ist mit Muscheln die Gestalt eines Drachen ausgelegt, zu seiner Linken, also im Westen, diejenige eines Tigers. Die Figuren beider Tiere liegen mit dem Rücken zur Leiche und weisen mit dem Kopf nach Norden. Zu Füßen der Leiche sind zwei Schenkel-

cf. Hentze, C.: Die Wanderung der Tiere um die heiligen Berge. In: Symbolon 4 (1964) 9-104, hier 14-25; id.: Funde in Altchina. Göttingen 1967, 200 sq.; Eberhard (wie not. 10) 253 sq.

Zitiert nach Schlegel, G.: Uranographie chinoise. La Haye 1875 (Nachdruck Milano 1977), 66.

26 Eberhard, W.: The Local Cultures of South and East China. Leiden 1968, 170 sq. In diesen Zusammenhang gehört vielleicht auch das Märchen num. 63 in Wilhelm (wie not. 2). Hier erscheint der Berggott zwar nicht als Tiger, wohl aber als Herr über die Toten.

28 Schlegel (wie not. 25) 39; Eberhard (wie not. 10) 352.

29 Bodde (wie not. 23) 129 sq. 
knochen quer hingelegt, dazu Muscheln, die ein Dreieck bilden. Die Bedeutung dieser Anordnung bleibt unklar. In einer kleinen Ausbuchtung östlich des Drachen liegen die Überreste eines Kindes oder Jugendlichen, schlecht erhalten und daher nicht genau zu bestimmen. Spiegelbildlich hierzu, in einer Ausbuchtung westlich des Tigers, ist ein etwa zwölfjähriges Mädchen bestattet; entsprechende Spuren am Schädel zeigen, daß es keines natürlichen Todes starb. Desgleichen findet sich in einer Ausbuchtung am Fußende des Grabes das Skelett eines etwa sechzehnjährigen Jungen; auch hier handelt es sich offensichtlich um ein Menschenopfer. Dies ist die älteste Bestattung der Yang-shao-Kultur, die eindeutig auf Totenfolge bzw. Menschenopfer hinweist ${ }^{30}$.

Was haben in diesem Zusammenhang die aus Muscheln geformten Figuren des Drachen im Osten, des Tigers im Westen zu bedeuten? Man sollte zunächst zur Kenntnis nehmen, daß dreitausend Jahre später dem 433 v. Chr. verstorbenen Markgrafen Yi von Tseng eine Kleidertruhe ins Grab mitgegeben wurde, auf deren Deckel wiederum Drache und Tiger aufgemalt sind, zwischen ihnen die Namen derjenigen 28 Sternbilder, die als ,Mondhäuser' gelten. Sie sind im Kreis um das Zeichen tou (Scheffel) gruppiert, d. h. um das Sternbild des Großen Bären einschließlich des Polarsterns ${ }^{31}$. Das Bild auf der Truhe repräsentiert demnach den gestirnten Himmel, ja den gesamten Kosmos gerade auch mit Hilfe der beiden Tiere, die ihrerseits - auch mit ihren Sternbildern - Osten und Westen symbolisieren. Der gesamte Kosmos soll der Überlieferung nach auch in dem (noch ungeöffneten) Grab des Ch'in Shih-huang-ti dargestellt sein, und eben dies, die symbolische Darstellung des Kosmos, nämlich der gestirnte Himmel und die Tiere der Vier Richtungen mit dem Drachen und dem Tiger auf der Ost- bzw. Westwand der Grabkammer, wurde selbst noch in dem gänzlich von chinesischen Ideen geprägten Takamatsu-Hügelgrab vom Ende des 7.Jahrhunderts in Japan gefunden ${ }^{32}$. Stets handelt es sich um denselben Gedanken, der in vereinfachter Weise bereits den beiden aus Muscheln gelegten Bildern von Drache und Tiger zugrunde liegt.

Warum aber hat man den Toten diese Darstellung in ihre letzte Ruhestätte mitgegeben? Auf das Wesentliche reduziert, d.h. in den Bildern von Tiger und Drache, den Symbolen von Herbst und Frühling, Tod und neuem Leben, kommt in voller Klarheit jener Gedanke zum Ausdruck, der zuvörderst den Toten in seinem Grabe bewegen muß, der Gedanke eines neuen Lebens in einer neuen

30 Sun Te-hsüan u.a.: Ho-nan P'u-yang Hsi-shui-p'o i-chih fa-chüeh chien-pao (Bericht über die Ausgrabungen in Hsi-shui-p'o, P'u-yang, Honan). In: Wen-wu (1988) H.3, $1-6$, hier 3 sq. Die Figur des Tigers ist 1,39 m lang, die des Drachen 1,78 m; cf. auch Brinker, H.: Vom Ursprung des Menschenbildes in der chinesischen Kunst. In: Das alte China. Menschen und Götter im Reich der Mitte, 5000 v. Chr. - 220 n. Chr. Redaktion R. Goepper. Ausstellungskatalog München 1995, 13-35, hier 19, mit Abbildung.

31 ibid.

32 Takamatsu-zuka hekiga-kan. Kaisetsu (Haus der Wandbilder des Takamatsu-Hügelgrabes. Erläuterungen). Nara 1980, 9-14. 
Welt. Garant dieses neuen Lebens sind die Gestirne mit ihrem Kommen und Gehen. Auf das Erscheinen des herbstlichen Sternbilds des Tigers, auf Untergang und Verfall folgt, sobald sich das Sternbild des Drachen am Himmel zeigt, ein neuer Frühling mit neuem Leben. Vor allem anderen aber ist es der Mond, der durch sein allmonatliches, jedermann sichtbares Sterben und Wiedererstehen den Gedanken von neuem Leben aus dem Tode vermittelt. So ist denn auch auf der erwähnten Truhe unter dem Bild des Tigers eine Axt mit eingerollten Enden aufgemalt, Symbol des Mondes oder des Mondumlaufs, Zeichen der ewigen Wiederkehr.

Noch weit bildhafter ersteht vor unseren Augen die Rolle des Tigers als Vernichter des Lebendigen in Darstellungen auf frühchinesischen Bronzegefäßen der Shang-Zeit, Gefäßen, die sämtlich im Totenkult Verwendung fanden und deren Symbolik sich deshalb in erster Linie auf den Tod und das Leben nach dem Tode bezieht. Diese Symbolik ist im 2. Jahrtausend v. Chr. voll ausgeprägt. Das Verdienst, ihren religiösen und kosmologischen Gehalt weitgehend erschlossen zu haben, gebührt Carl Hentze.

Die älteste anhand der Shang-Bronzen erschlossene Vorstellung ist die des Tigers als t'ao-t'ieh (Fresser), eine wandelbare Gottheit, die mit verschiedenen Attributen auftritt, zu denen Büffelhörner und Flügel zählen, jedoch immer von der Tigermaske ausgehend. Er gehört zur Erde; ja, er ist nicht nur Symbol der Unterwelt, der Tigerrachen selbst ist Ein- und Ausgang zur Unterwelt, zum Erdinnern, zur Welt der Toten. Doch der t'ao-t'ieh, der ,Fresser', der unter dem Bilde des Tigers auftritt, ist keineswegs nur eine Todesgottheit. Der Verschlinger ist gleichzeitig ein Herauswürger, der Vernichter ein Lebensspender ${ }^{33}$.

Die Ambivalenz des Tigers läßt sich am einzelnen Kultgefäß wie an zusammengehörigen Gefäßgruppen ablesen. So erkennt man etwa an einer zusammengehörigen Gruppe von drei sogenannten Ho-Gefäßen, übereinandergestaffelt, den Verlauf der Geschehnisse: Oben, an der Scheitelpartie befindet sich jedesmal die verschlingende Maske mit einem Maule, das gleichzeitig der Eingang für den Geist des Toten ist. Unten am Fuß der Gefäße ist die große befreiende Maske angebracht, aus deren Rachen die Zikade hervorkommt: Zeichen der Wiedergeburt aus der Erde. Zwischen diesen Extremen geht die Totenreise vor sich, die erst zur Unterwelt und Dunkelheit, dann wieder zu deren Verlassen und zum Lichte nach oben führt. Es sind auch hier zunächst kosmische Gegensätze, die in der feststehenden Ikonographie dieser Gefäße zum Ausdruck gebracht werden, Aufgang und Untergang, Tod und Erneuerung, erdacht in Verbindung mit den Erscheinungen des Himmels, doch diese werden auf das Leben des Menschen projiziert. Der Eingang durch den Rachen der großen Masken ist der Eingang zu Nacht und Dunkelheit, er bedeutet das Sterben, in dem sich das Menschenge-

33 Hentze, C.: Bronzegerät, Kultbauten, Religion im ältesten China der Shang-Zeit. Antwerpen 1951, 102, 104, 168; id.: Die Tierverkleidung in Erneuerungs- und Initiationsmysterien. In: Symbolon 1 (1958) 39-86, hier 73 sq.; id. 1964 (wie not. 24) 31-34; id. 1967 (wie not. 24) 132-135, 170-174, 181, 200-202. 
schick vollzieht, das der Gestirnsevolution gleichgesetzt wird. Dem Tiger kommt hierbei die Doppelfunktion der Tötung und der Zeugung zu. In Verbindung mit dem Urahnen, den er in anderen Darstellungen in den Armen hält, zeigt er sich in seiner vollen mythischen Bedeutung: als Vertreter der Erdgottheit, die den ersten sterblichen Menschen erstehen läßt ${ }^{34}$.

Der t'ao-t'ieh oder der Tiger galt demnach ursprünglich als ein ambivalentes Wesen, doch in den Jahrhunderten um Christi Geburt ging dieser Doppelaspekt offenbar verloren. Geblieben ist die bedrohliche Seite, die des Todesdämons, wie sie sich in den Wer-Tigern der Sage und schließlich in der Märchenfigur des menschenfressenden Tigerdämons manifestiert. Und dennoch scheint noch ein schwacher Nachhall des Leben spendenden Tigers gegenwärtig, wenn von Opferplätzen für den ,Ehevermittler Tiger ' die Rede ist, wenn der dankbare Tiger eine Schwiegertochter herbeischleppt oder die Tigermutter ein Menschenkind aufzieht.

34 id. 1951 (wie not. 33) 156-158; id. 1967 (wie not. 24) 174-206. 